\title{
Multivariate study of spectral data of oils
}

\author{
Hana Vaskova ${ }^{1, *}$, and Martina Buckova $^{2}$ \\ ${ }^{1}$ Tomas Bata University in Zlin, Faculty of Applied informatics, Department of Electronic and Measurements, Zlin, \\ Czech Republic \\ ${ }^{2}$ Tomas Bata University in Zlin, Faculty of Technology, Department of Food Technology, 76001 Zlin, Czech Republic
}

\begin{abstract}
In this paper the study of structural differences and thermal degradation of edible oils during heating is presented. The study is performed on five types of vegetable oil: extra virgin olive oil, pomace olive oil, sunflower, canola and palm oil. The oils were measured by Raman spectroscopy. This method brings advantages as rapidity, independence on chemicals, provides specific information on chemical composition and structure of material and is able to detect structural changes. For the processing of large data sets multivariate analytical method as Principal component analysis and Cluster analysis are applied to find the patterns within the data. The evaluation of process of thermal degradation is also based on the major decomposition product of oxidized linoleate appearing in spectra via band $1640 \mathrm{~cm}^{-1}$. Mathematically processed data indicate the least effect of heating for olive oils, the greatest degradation and loss of unsaturation for sunflower oil.
\end{abstract}

\section{Introduction}

Edible oils are commonly used for preparing many kinds of food and high temperatures are often applied. It is already known that extra virgin oils are more suitable for preparing "cold" meals. Others, mainly refined, are suitable for frying or baking at higher temperature. However, even the edible oils that are relatively thermal resistant should be controlled because of formation of oxidation products. Therefore, the thermal stability of edible oils is a topic of research studies using various methods.

The conventional analytical methods for edible oils quality determination require pre-treatment of samples, reagents and solvents using as well as lab equipment and skills of the lab technicians. That is why new rapid and simple methods are still demanded. Raman spectroscopy seems to be very promising technique combining rapid and relatively simple approach with sensitivity for detection of changes in molecular structure. Moreover, Raman spectra can be obtained via transparent glass or plastic vials and this measurement can be done as on-line analysis and/or by portable Raman instruments.

The objective of this study is to compare different types of edible oils and to explore their thermal stability using Raman spectroscopy. Methods that enable multivariate analysis such as Principal component analysis and Cluster analysis are used for data mining from the large spectral data sets.

\section{Vegetable oils}

Vegetable oils consist of triglycerides in amount of $95-98 \%$. The rest is formed by minor compounds as carotenes, tocopherols, sterols, squalene and free acids $[1,2]$. The triglycerides are composed of fatty acids (FA) with characteristic number of carbon atoms in chain and amount of saturated or unsaturated bonds that are responsible for behaviour of edible oils. The main saturated fatty acids in vegetable oils are considered palmitic acid (C16:0) and stearic acid (C18:0). Oleic acid is the major monounsaturated fatty acid (C18:1). Linoleic acid (C18:2) and linolenic acid (C18:3) are the major polyunsaturated fatty acids. Each type of vegetable oil is characterized by its own specific fatty acids ratio content $[2,3]$.

Lipids are important and irreplaceable part of the human diet as well as proteins and carbohydrates. The role of lipids in organism is essential. Hence the intake of vegetable oils is very important. Except saturated fatty acids they also contain a substantial proportion of monounsaturated and polyunsaturated fatty acids. The unsaturated fatty acids represent health benefit of edible oils in their consumption, however, also a source of instability. Vegetable oils undergo oxidative degradation that can be caused during storage, technological operation in food production or during frying.

\subsection{Thermal degradation}

Thermal stress speeds up oxidative processes concerning predominantly polyunsaturated fatty acids as linoleic and linolenic acids according to high double bonds content. The major oxidation products are esters, alcohols, aldehydes, ketones, lactones and hydroxy compounds. Presence of these products strongly affects quality of edible oils (taste and aroma) and their nutritional value.

* Corresponding author: author@e-mail.org 
Moreover, many of oxidation products are considered as potentially toxic in relatively low concentration [4]. According to this health risk is necessary to detect formation of oxidative products during oil heating. Some of qualitative changes of vegetable oils can be reflected in Raman spectra.

\section{Materials and methods}

Raman spectroscopy was used for the study of characteristics of oils and changes occurring during thermal degradation of oils. Obtained spectra were evaluated using multivariate analytical methods Principal component analysis and Cluster analysis.

\subsection{Materials}

Five different types of edible oils, four refined and one extra-virgin were used for spectroscopic analysis: extravirgin olive oil, olive pomace oil, canola, sunflower and palm. All of these oils were purchased from common markets.

At the beginning these oils were measured at lab temperature, later heated for up to $160 \pm 5{ }^{\circ} \mathrm{C}$ and the temperature was maintained for 5 hours to monitor the thermal degradation process. The amount of 2 litres of each of oils was heated up The first sample of $100 \mathrm{ml}$ was taken right after reaching the temperature of $160{ }^{\circ} \mathrm{C}$ other samples after every 30 minutes in the same amount. Raman microscopy measurement followed.

\subsection{Raman spectroscopy and instrumentation}

Raman spectroscopy provides very specific chemical „fingerprint“ of every single chemical substance in the form of the Raman spectrum. In other words Raman spectroscopy reflects chemical composition and structure of materials what makes the method proper for material identification and study of structural changes. Moreover, considering the advantages of the method, Raman spectroscopy becomes popular and valuable part of laboratories around the world in recent years.

The method is based on Raman scattering - an inelastic scattering resulting from an interaction of a photon and a molecule. Photons of inelastic scattering have slightly changed wavelengths (compared to incident laser photons), that are characteristic for specific bonds in surveyed material. Since most photons are on molecules scattered elastically (Rayleigh scattering without changing the wavelength), it is necessary to filter out of the spectrum of the strongly present wavelength of laser.

Raman spectroscopy brings many advantages as the method is relatively rapid, non-destructive, contactless, usable for measuring through transparent glass or polymeric covering layers or containers, applicable to all states of matter and different forms, without special requirements for sample preparation, usable as in situ analysis. The most often appearing drawback of the method is the fact that Raman scattering is a weak effect.
Luminescence as much stronger quantum effect with bigger intensity can overlap Raman spectra and mask spectral information $[5,6]$.

For the measurement, inVia Basis Raman microscope form Renishaw was used. Raman instrument uses two lasers as light sources: argon ion laser with the excitation wavelength $514 \mathrm{~nm}$ and maximum output power of 20 $\mathrm{mW}$ and $785 \mathrm{~nm}$ NIR diode laser with maximum output power $300 \mathrm{~mW}$. Both were tested but more precise results were obtained using NIR laser.

A Leica DM 2500 confocal microscope with the resolution $2 \mu \mathrm{m}$ was coupled to the Raman spectrometer. All measurements were collected with $20 \mathrm{~s}$ exposure time and 5 accumulations. The samples were firstly scanned in range 100 to $3200 \mathrm{~cm}^{-1}$ with $1 \mathrm{~cm}^{-1}$ spectral resolution. After determining the principle peaks the spectral range was reduced approximately to the area $800-1800 \mathrm{~cm}^{-1}$.

\subsection{Principal component analysis}

Raman spectral data are multivariate, since they reflect the composition of material and its structure. Raman peaks are assigned to the vibrations of chemical bonds in surveyed materials. For the evaluation it is advantageous to use multivariate mathematics.

Principal Component Analysis (PCA) is useful and powerful statistical method for analysing data. PCA finds application in various fields from chemistry via neuroscience to computer face recognition, image compression or social studies. Basically it finds and identifies patterns in datasets of high dimension and highlights hidden similarities and differences. It extracts relevant information from confusing data sets finding new variables for creation better imaginable mathematical space.

\subsection{Cluster analysis}

Cluster analysis (CA) is an explorative analysis offering patterns or structures recognition within the data. The method divides data into groups (clusters) in such a way, that objects in the same group are more similar to each other than to objects from other clusters.

\section{Results and discussion}

Raman spectral data of 5 different oils exhibit similar distribution of characteristic peaks due to their composition. However, each of oils has various ratios of saturated (SFA), monounsaturated (MUFA), polyunsaturated fatty acids (PUFA) and other components, what affects intensity and Raman shift of bands in spectra. Raman spectra of 5 oils are displayed in Fig. 1. The linear baseline correction and normalization according $1747 \mathrm{~cm}^{-1}$ band was applied. Raman band $1747 \mathrm{~cm}^{-1}(\mathrm{C}=\mathrm{O}$ ester carbonyl) was taken as an internal standard for normalization because it does not exhibit any changes in the structure. Essential molecular assignments for the measured spectral range 


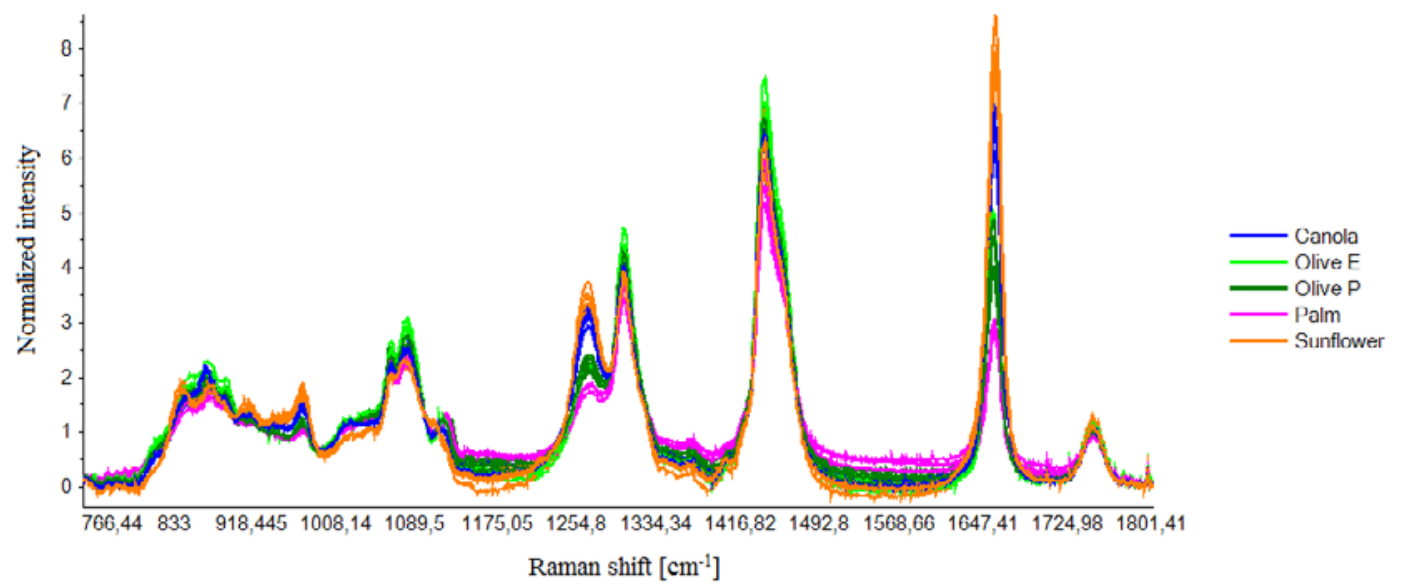

Fig. 1. Raman spectra of five types of vegetable oil, normalized.

are listed in Table 1 and are in accordance with literature $[7,8]$.

The cluster analysis was performed for the Raman spectra of 5 cold (room temperature before heating) oils. The hierarchical complete linkage based on correlation distance measure was used. There is the dendrogram in Fig. 2 of spectral area $1200-1390 \mathrm{~cm}^{-1}$, which refers to bands $1267 \mathrm{~cm}^{-1}$ and $1303 \mathrm{~cm}^{-1}$. Intensities of these bands reflect the amounts of saturated and unsaturated fatty acids with straight aliphatic chains and predominantly with 16 or 18 number of carbon atoms in chains. Various types of oils differ in the proportion of these components, what can be used for their differentiation.

The relative intensity ratio of the bands at $1265 \mathrm{~cm}^{-1}$ and $1303 \mathrm{~cm}^{-1}$ is usually used to determine the degree of unsaturation in the oil [9]. Two olive oils are the most similar. This group is enlarged by palm oil what can be affected by the similar slight higher content of palmitic and stearic acids. Second group consist of sunflower and canola oil. In comparison to the first group, sunflower and canola oils have rather higher amounts polyunsaturated fatty acids, mainly linoleic acid.

The context of saturated and unsaturated FA in oils is clearly visible also in the spectra in Fig. 1. for bands $1267 \mathrm{~cm}^{-1}$ and $1658 \mathrm{~cm}^{-1}$ (saturated) and $1303 \mathrm{~cm}^{-1}$ and $1442 \mathrm{~cm}^{-1}$ (unsaturated FA). Fig. 3 represents the dendrogram of spectral area1200 $-1800 \mathrm{~cm}^{-1}$. Here, the

Table 1 Assignments for Raman bands.

\begin{tabular}{|c|c|}
\hline $\begin{array}{c}\text { Raman shift } \\
{\left[\mathrm{cm}^{-1}\right]}\end{array}$ & Molecular vibration \\
\hline 1267 & $=\mathrm{C}-\mathrm{H}$ symmetric rocking \\
\hline 1303 & $\mathrm{CH}_{2}$ in-plane twist \\
\hline 1442 & $\mathrm{CH}_{2}$ scissoring \\
\hline 1526 & $\begin{array}{c}\mathrm{C}=\mathrm{C} \text { stretching of carotenoids } \\
\text { (extra virgin oils) }\end{array}$ \\
\hline 1640 & $\mathrm{C}=\mathrm{C}$ trans, trans 2,4 decadienal \\
\hline 1658 & $\mathrm{C}=\mathrm{C}$ cis double bond stretching \\
\hline 1747 & $\mathrm{C}=\mathrm{O}$ ester-carbonyl stretching \\
\hline
\end{tabular}

layout within clusters is alike, but the difference is in worse correlation, especially for the connection of palm oil to the olive oils. In this area spectral characteristic are more complex what affects the pattern creation.

Thermal degradation was studied on all heated samples. In Raman spectra can be seen formation of the degradation product at $1640 \mathrm{~cm}^{-1}$ assigned to trans, trans-2,4-decadienal. It is one of the oxidative products. to the $\mathrm{C}=\mathrm{C}$ stretching vibration in conjugated system [10]. The dependence of normalized intensities of band $1640 \mathrm{~cm}^{-1}$ on time is shown in Fig. 4. Intensities were acquired from fitting using normalization via $1747 \mathrm{~cm}^{-1}$ band and subtraction of the intensity at the room

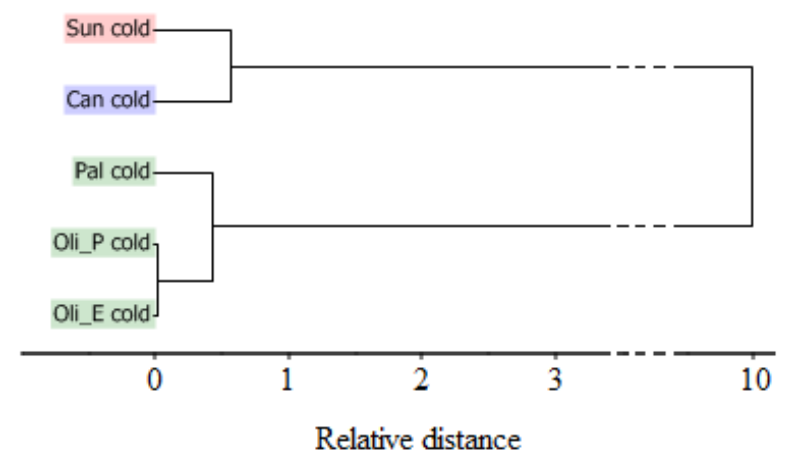

Fig. 2. Cluster analysis of cold oils in area $1200-1390 \mathrm{~cm}^{-1}$.

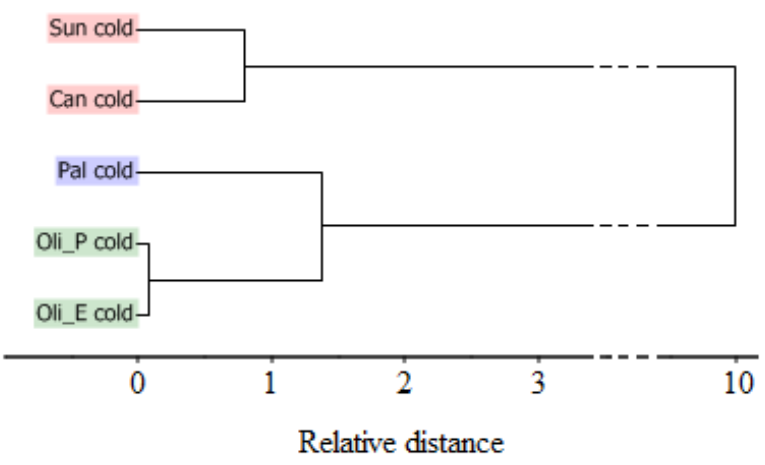

Fig. 3. Cluster analysis of cold oils in area $1200-1800 \mathrm{~cm}^{-1}$. 


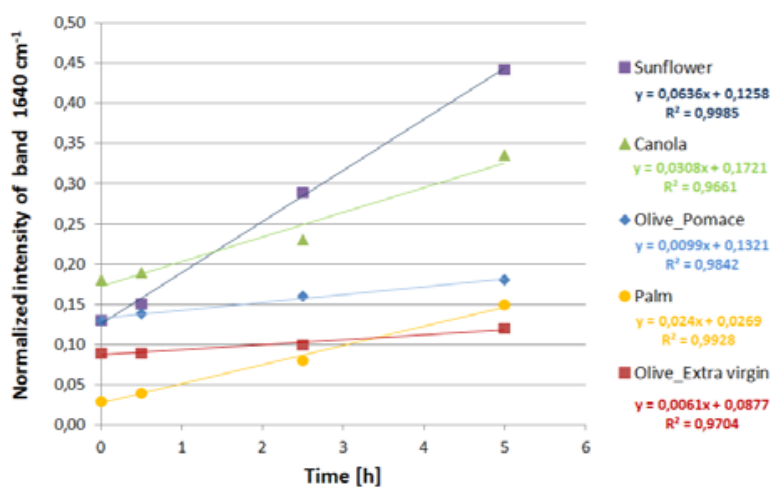

Fig. 4. Thermal degradation of edible oils according to normalized intensities of Raman band at $1640 \mathrm{~cm}^{-1}$.

temperature. Results show the smallest structural changes for extra virgin olive oil followed by pomace olive oil. The biggest impact of oxidative change is observed for sunflower oil.

For the clarity of the outputs of multivariate analysis cold and heated oils for 2.5 hours and 5 hours were used. The dendrogram for named samples and the whole measured spectral area is displayed in Fig. 5. The first

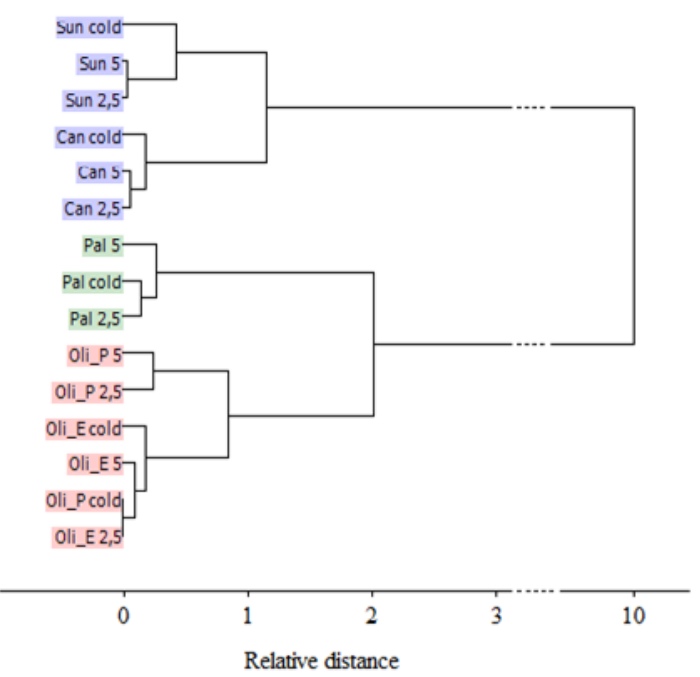

Fig. 5. Cluster analysis of cold and heated oils.

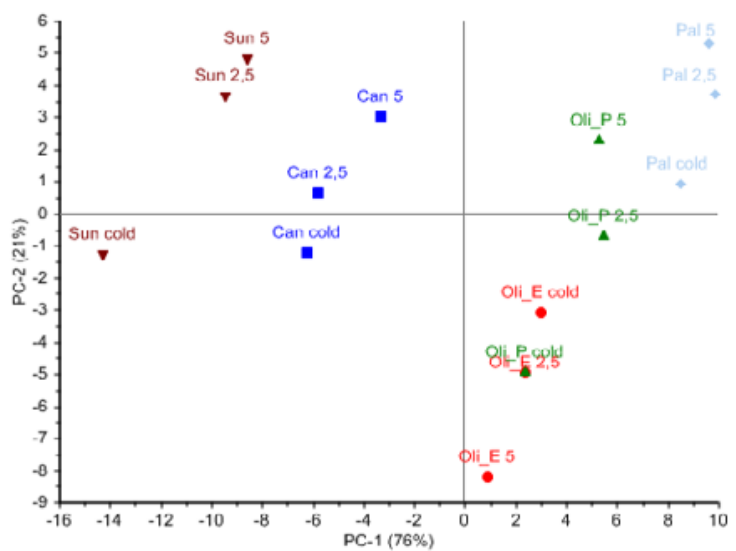

Fig. 6. PCA scores of cold and heated oils. cluster consists of sunflower and canola oils which create clear sub-clusters with diversity of cold and heated samples. Second cluster consists of two subclusters of palm oil samples and olive oils.

Spectral data of 5 cold and thermally degraded samples were used also for the PCA. The result is shown in Fig. 6. The diversity is sharply distinguished from the other species of oils. The only one overlap appears for extra virgin and pomace olive oils, what supports the closeness to their composition over other oils.

All 4 oils except of extra virgin olive oil show the same trends according to growing PC-1 and PC-2. This only one - extra virgin oil has the opposite trend.

\section{Conclusions}

Data reflecting structural variations of 5 types of edible oils were acquired by modern spectroscopic method Raman spectroscopy. Multivariate statistical methods Cluster analysis and Principal component analysis were used for the evaluation of the datasets. The results show good agreement with the known ratio of saturated and unsaturated fatty acids in the oils. The assessment of thermal degradation can be done via amount of arising degradation products as trans, trans-2,4-decadienal. The most evident degradation was observed for sunflower oil, then for canola and palm oil. The best thermal stability show extra virgin olive oil followed by pomace olive oil. However, the thermal stress speeds up undesired decomposition of vegetable oils and promotes creation of unsafe and unhealthy oxidative products in all types of tested oils. This oxidative process is more intensive with the longer period of heating.

This work was supported by the Ministry of Education, Youth and Sports of the Czech Republic within the National Sustainability Programme project No. LO1303 (MSMT7778/2014) and also by the European Regional Development Fund under the project CEBIA-Tech No. CZ.1.05/2.1.00/03.0089.

\section{References}

1. Y. G. M. Konbonga, H. Ghalila, et al, F. N. S., 02, (2011)

2. Frank D. Gunstone, Vegetable Oils in Food Technology: Composition, Properties and Uses (Wiley-Blackwell, 2011)

3. R. C. Zambiazi, R. Przybylski, M. W. Zambiazi, C. B. Mendonca, CEPPA, Curitiba 25, 1 (2007)

4. S. A. Smith, Food Chem. 102 (2007)

5. N. B. Colthup, H. D. Lawrence, S. E. Wiberley, Introduction to infrared and Raman spectroscopy, (1990)

6. R. S. Das, Y. K. Agraval, Vibr. Spec. 57, 2 (2011)

7. S. Galier, K.C. Gordon, R. Jimenez-Flores, D.W. Everett, Int. Dairy J. 21, 6 (2011)

8. B. Muik, B. Lendl, A. Molina-Diaz, M.J. Ayora-Canada, Appl. Spectrosc. 57, 2 (2003)

9. E.C.Y. Li-Chan, Trends Food Sci. Tech. 7 (1996)

10. B. Muik, B. Lendl, A. Molina-Diaz, M.J. Ayora-Canada, Chem. Phys. Lipids 134 (2005) 